\title{
Editorial
}

\section{Mathematical Problems for Complex Networks}

\author{
Zidong Wang, ${ }^{1,2}$ Jinling Liang, ${ }^{3}$ and Yurong Liu ${ }^{4}$ \\ ${ }^{1}$ School of Information Sciences and Technology, Donghua University, Shanghai 200051, China \\ ${ }^{2}$ Department of Information Systems and Computing, Brunel University, Uxbridge, \\ Middlesex UB8 3PH, UK \\ ${ }^{3}$ Department of Mathematics, Southeast University, Nanjing 210096, China \\ ${ }^{4}$ Department of Mathematics, Yangzhou University, Yangzhou 225002, China
}

Correspondence should be addressed to Zidong Wang, zidong.wang@brunel.ac.uk

Received 16 November 2011; Accepted 16 November 2011

Copyright (C) 2012 Zidong Wang et al. This is an open access article distributed under the Creative Commons Attribution License, which permits unrestricted use, distribution, and reproduction in any medium, provided the original work is properly cited.

Complex networks do exist in our lives. The brain is a neural network. The global economy is a network of national economies. Computer viruses routinely spread through the Internet. Food-webs, ecosystems, and metabolic pathways can be represented by networks. Energy is distributed through transportation networks in living organisms, man-made infrastructures, and other physical systems. Dynamic behaviors of complex networks, such as stability, periodic oscillation, bifurcation, or even chaos, are ubiquitous in the real world and often reconfigurable. Networks have been studied in the context of dynamical systems in a range of disciplines. However, until recently there has been relatively little work that treats dynamics as a function of network structure, where the states of both the nodes and the edges can change, and the topology of the network itself often evolves in time. Some major problems have not been fully investigated, such as the behavior of stability, synchronization and chaos control for complex networks, as well as their applications in, for example, communication and bioinformatics.

Complex networks have already become an ideal research area for control engineers, mathematicians, computer scientists, and biologists to manage, analyze, and interpret functional information from real-world networks. Sophisticated computer system theories and computing algorithms have been exploited or emerged in the general area of computer mathematics, such as analysis of algorithms, artificial intelligence, automata, computational complexity, computer security, concurrency and parallelism, data structures, knowledge discovery, DNA and quantum computing, randomization, semantics, symbol manipulation, numerical analysis, and mathematical software. This special issue aims to bring together the latest approaches to understanding complex networks from a dynamic system perspective. Topics include, but are not limited to the following aspects of dynamics analysis for complex 
networks: (1) synchronization and control; (2) topology structure and dynamics; (3) stability analysis; (4) robustness and fragility.

This special issue aims to bring together the latest approaches to understanding the mathematical issues of complex networks from a dynamic system perspective. We have solicited submissions to this special issue from electrical engineers, control engineers, mathematicians, and computer scientists. After a rigorous peer-review process, 21 papers have been selected that provide overviews, solutions, or early promises, to manage, analyze, and interpret dynamical behaviors of complex networks. These papers have covered both the practical and theoretical aspects of complex networks in the broad areas of dynamical systems, mathematics, statistics, operational research, and engineering.

This special issue starts with a survey paper on the recent advances of filtering and control for complex networked systems with incomplete information. Specifically, in the paper entitled "Recent advances on filtering and control for nonlinear stochastic complex systems with incomplete information" by $\mathrm{Z}$. Wang, the focus is mainly on the filtering and control problem for complex systems with incomplete information and the main aim is to give a survey on some recent advances in this area. The incomplete information under consideration includes missing measurements, randomly varying sensor delays, signal quantization, sensor saturations, and signal sampling. The modeling issues are first discussed to reflect the real complexity of the nonlinear stochastic systems. Based on the models established, various filtering and control problems with incomplete information are reviewed in detail. Then, the complex systems, are dealt with from three aspects, that is, nonlinear stochastic systems, complex networks and sensor networks. Both theories and techniques for dealing with complex systems are reviewed and, at the same time, some challenging issues for future research are raised. Subsequently, the filtering problems for the stochastic nonlinear complex networks with incomplete information are paid particular attention by summarizing the latest results. Finally, some conclusions are drawn and several possible related research directions are pointed out.

Complex networks are composed of a large number of highly interconnected dynamical units and therefore exhibit very complicate dynamics. Examples of such complex networks include the Internet, which is a network of routers domains, the World Wide Web, which is a network of web sites, the brain, which is a network of neurons, and an organization, which is a network of people. Synchronization for complex networks is attracting more and more research attention due to its ubiquity in many system models of the natural world. In another paper "Impulsive synchronization of nonlinearly-coupled complex networks" by J. Cao, the impulsive synchronization problem is investigated for nonlinearly coupled complex networks. Based on the stability analysis of impulsive functional differential equations, some sufficient synchronization criteria are established in terms of average impulsive interval. The model addressed is a nonlinearly coupled network that covers the linearly coupled network and an array of linearly coupled systems as special cases. In the work "Enhancement of the quality and robustness in synchronization of nonlinear lur'e dynamical networks" by Y. Yang, the synchronization is studied for a class of nonlinear dynamical networks with faults and external disturbances. Sufficient conditions are given to guarantee the global robust synchronization for the network by means of solving the linear matrix inequalities. By using adaptive-impulsive control approach, the projective synchronization problem is dealt with in "Adaptive-impulsive control of the projective synchronization in driveresponse complex dynamical networks with time-varying coupling" by S. Zheng for drive-response time-varying coupling complex dynamical networks with time delay and time-varying weight links. An adaptive feedback controller with impulsive control effects is designed. In 
the paper entitled "Second-order consensus for multi-agent systems under directed and switching topologies" by L. Gao, based on the graph theory and Lypunov method, sufficient conditions of the consensus stability are established for systems with neighbor-based feedback laws in leader-following case and leaderless case. As special cases, the consensus criteria for balanced and undirected interconnection topology cases can be readily established.

Sensor networks have recently been undergoing a quiet revolution in all aspects of the hardware implementation, software development, and theoretical research. Sensor networks possess their own characteristics due mainly to the large number of inexpensive wireless devices (nodes) densely distributed and loosely coupled over the region of interest. The past decade has seen successful applications of sensor networks in many practical areas ranging from military sensing, physical security, air traffic control, to industrial and manufacturing automation. In the paper addressed "Energy-aware topology evolution model with link and node deletion in wireless sensor networks" by X. Luo, based on the complex network theory, a new topological evolving model is proposed. In the evolution of the topology of sensor networks, the energy-aware mechanism is taken into account, and the phenomenon of change of the link and node in the network is discussed. Theoretical results and numerical simulation are given to analyze the topology characteristics and network performance with different node energy distributions. It is shown that, when nodes energy is more heterogeneous, the network is better clustered and the higher performance is achieved in terms of the network efficiency and the average path length of transmitting data. In order to maintain $k$ disjoint communication paths from source sensors to the macronodes, a hybrid routing scheme is developed in "An immune cooperative particle swarm optimization algorithm for fault-tolerant routing optimization in heterogeneous wireless sensor networks" by Y.-S. Ding, where multiple paths are calculated and maintained in advance, and alternative paths are created on demand. Also, an immune cooperative particle swarm optimization algorithm (ICPSOA) is developed to guarantee the fast routing recovery and reconstruct the network topology from path failure in heterogeneous wireless sensor networks (H-WSNs). In another paper "Geometric buildup algorithms for sensor network localization" by $\mathrm{Z}$. Wu, a geometric build-up algorithm is given for the sensor network localization problem with either accurate or noisy distance data. Moreover, an algorithm with two buildup phases is presented to handle the noisy and sparse distance data. By comparing with the existing approaches, the advantages of the proposed algorithms are shown.

In the past few decades, neural networks have received considerable research interests and have found successful applications in a variety of areas such as pattern recognition, associative memory, and combinatorial optimization. The dynamical characteristics of neural networks with time delays have become a subject of intense research activities. In the paper entitled "Global robust stability of switched interval neural networks with discrete and distributed time-varying delays of neural type" by $\mathrm{H}$. Wu, a switched interval neural network is discussed with discrete and distributed time-varying delays. Together with the Lyapunov approach and linear matrix inequality (LMI) technique, a delay-dependent criterion is given such that the switched interval neural network is globally asymptotically stable. By constructing the Lyapunov-Krasovskii functional and using the reciprocal convex technique, a new sufficient condition is derived in "Further stability criterion on delayed recurrent neural networks based on reciprocal convex technique," by $\mathrm{T}$. Li to guarantee the global stability for recurrent neural networks with both time-varying and continuously distributed delays. Numerical examples are given to show the effectiveness and less conservatism of the proposed method. In another paper " $H_{\infty}$ Neural-network-based discrete-time fuzzy control of continuous-time nonlinear systems with dither," by J.-D. Hwang, and by constructing a discrete-time (DT) fuzzy controller, the 
stabilization problem is investigated for a class of continuous-time (CT) nonlinear systems. After discretizing the CT nonlinear system, a neural-network (NN) system is established to approximate the DT nonlinear system. Then, a Takagi-Sugeno DT fuzzy controller is designed to stabilize this NN system. It is shown that when the discretized frequency or sampling frequency of the CT system is sufficiently high, the DT system can maintain the dynamic of the original CT system. Moreover, the trajectory of the DT system and the CT system can be made as close as desired by designing the sampling frequency.

As being well known, the cyber world brings massive changes to the society. There have been many cyber-related challenging problems that arise inevitably and should be handled. In the paper addressed "Two quarantine models on the attack of malicious objects in computer network," by B. K. Mishra, SEIQR (susceptible, exposed, infectious, quarantined, and recovered) models for the transmission of malicious objects are discussed in computer network with simple mass action incidence and standard incidence rate. Sufficient conditions for global stability and asymptotic stability of endemic equilibrium are given for simple mass action incidence. Also, the behaviors are analyzed for the susceptible, exposed, infected, quarantined, and recovered nodes in the computer network. The spatially embedded networks are tackled in "Structural models of cortical networks with long-range connectivity," by S. Rotter with specific distance-dependent connectivity profiles. By applying the stochastic graph theory, the structure and the topology of such networks are considered. In another paper "Abstract description of internet traffic of generalized cauchy type," by M. $\mathrm{Li}$, the set-valued analyses are investigated for the traffic of the fractional Gaussian noise (fGn) type and the generalized Cauchy (GC) type. Meanwhile, a design procedure of the autocorrelation function (ACF) is presented for the GC process in Hilbert spaces. Multiple complex tasks commonly occur in the water distribution networks, such as design, planning, operation, maintenance, and management. In the paper entitled "On the complexities of the design of water distribution networks," by J. Izquierdo, a synergetic association between swarm intelligence and multiagent systems is discussed for water distribution networks, where human interaction is simultaneously enabled. In the paper addressed "A novel algorithm of stochastic chance-constrained linear programming and its application," by C. Wang, the stochastic chance-constrained linear programming problem is investigated. A simplex algorithm is developed based on the stochastic simulation and a practical example is presented to illustrate the applicability of the proposed algorithm.

Stability analysis and stabilization problems of stochastic systems have attracted much attention in the past decades, since stochastic modeling has come to play an important role in many real-world systems, including nuclear, thermal, chemical processes, biology, socioeconomics, immunology, and so forth. In another paper "Mixed $\mathrm{H}_{2} / \mathrm{H}_{\infty}$ performance analysis and state-feedback control design for networked systems with fading communication channels," by A.-M. Stoica, the aim is to develop a performance analysis approach for networked systems with fading communication channels. A state feedback controller is designed to accomplish a mixed $H_{2} / H_{\infty}$ performance requirement. A numerical iterative procedure is presented which can be utilized to compute the stabilizing solution for system with jumps. The practical stabilization problem is investigated in "The practical stabilization for a class of networked systems with actuator saturation and input additive disturbances," by D. Chen for a class of linear systems with actuator saturation and input additive disturbances. The time-invariant and time-varying input additive disturbances are considered, respectively. By applying the Riccati equation approach and designing the linear state feedback controllers, sufficient conditions are established to guarantee the semiglobal practical stabilization for the closed-loop systems. In the paper entitled "A quasi-ARX model for multivariable decoupling 
control of nonlinear MIMO system," by J. Hu, a multiinput and multioutput (MIMO) quasi-autoregressive exogenous (ARX) model is presented and a multivariable-decoupling proportional integral differential (PID) controller is designed for the MIMO nonlinear systems. An adaptive control algorithm is given by using the MIMO quasi-ARX radial basis function network (RBFN) prediction model. In another paper "Practical stability in the pth mean for Itô stochastic differential equations," by H. Shu, the $p$ th mean practical stability problem is investigated for a general class of Itô-type stochastic differential equations over both finitetime and infinite-time horizons. Sufficient conditions are established such that the addressed differential equations are $p$ th moment practically stable. The practical stability problem is studied in "pth mean practical stability for large-scale Itô stochastic systems with markovian switching," by H. Shu for Markovian switching systems in the pth mean sense. By using the Lyapunov method, some criteria are presented such that various types of practical stability in the $p$ th mean are guaranteed for the nonlinear stochastic systems.

\section{Acknowledgments}

This special issue is a timely reflection of the research progress in the area of mathematical problems for complex networks. We would like to acknowledge all authors for their efforts in submitting high-quality papers. We are also very grateful to the reviewers for their thorough and ontime reviews of the papers.

Zidong Wang

Jinling Liang

Yurong Liu 


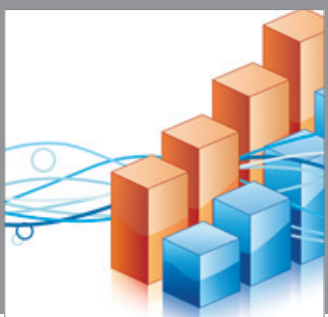

Advances in

Operations Research

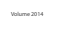

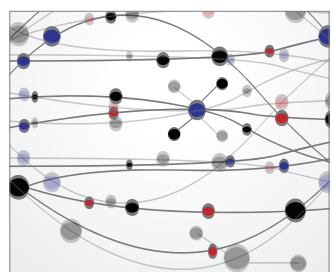

\section{The Scientific} World Journal
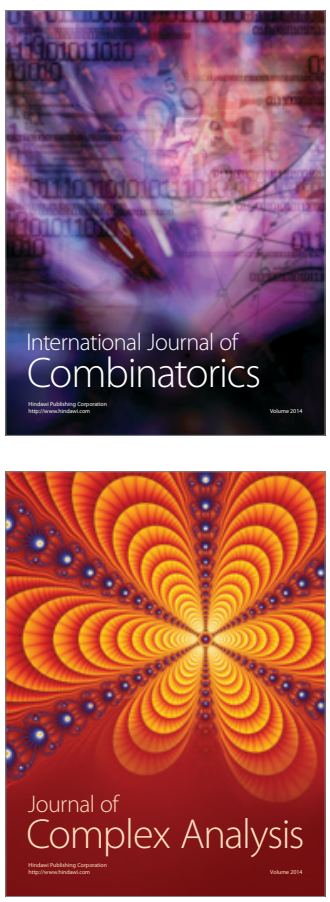

International Journal of

Mathematics and

Mathematical

Sciences
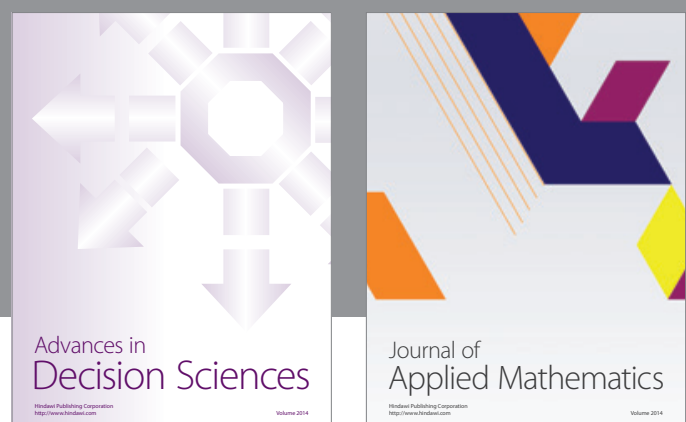

Journal of

Applied Mathematics
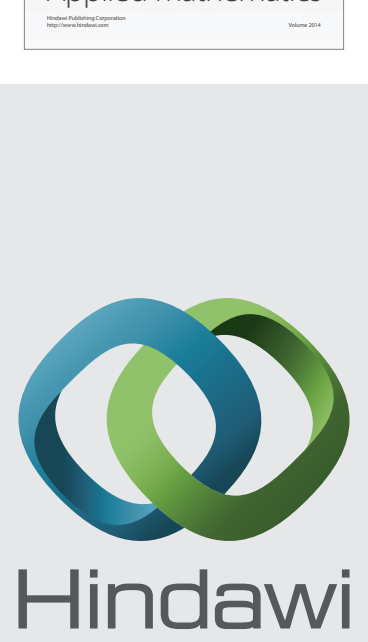

Submit your manuscripts at http://www.hindawi.com
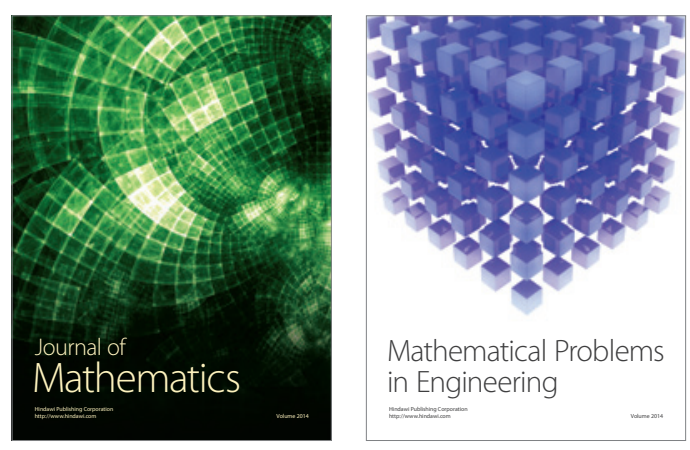

Mathematical Problems in Engineering
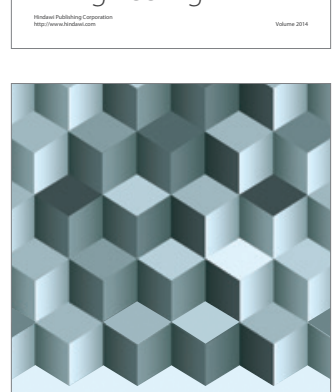

Journal of

Function Spaces
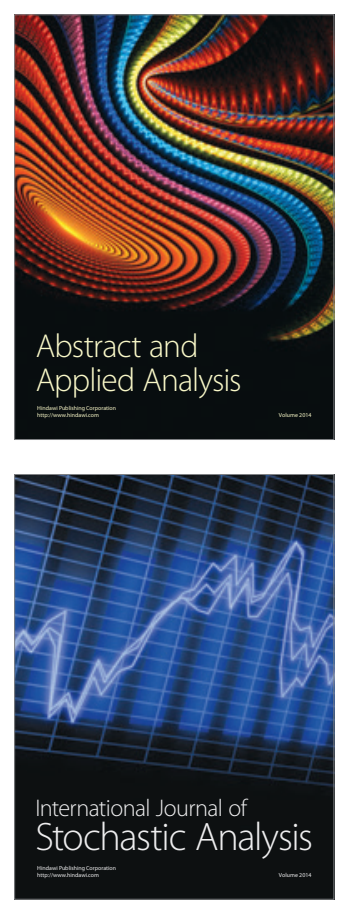

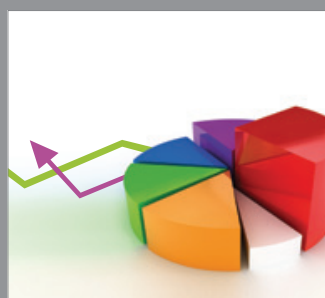

ournal of

Probability and Statistics

Promensencen
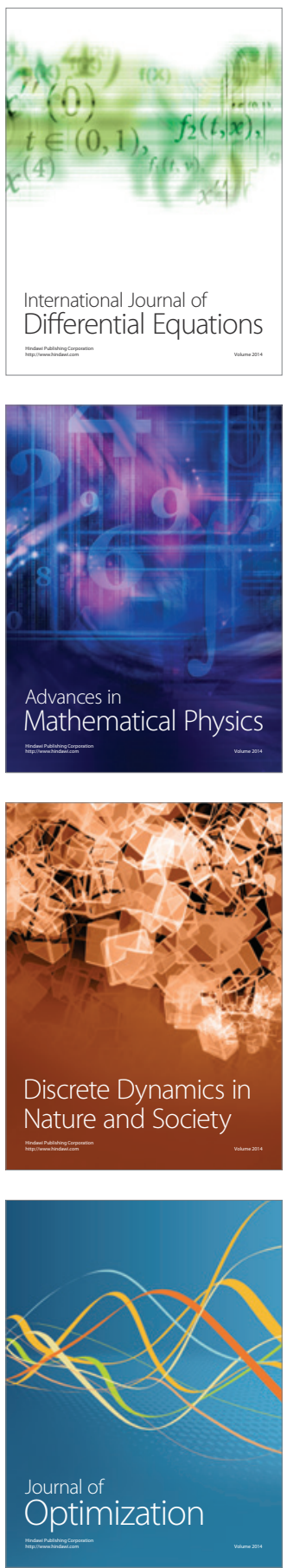